\title{
Heat shock modulates the expression of sirtuins and var genes in the malaria parasite Plasmodium falciparum
}

\author{
Linda Anagu \\ Keele University \\ David Hulse \\ Keele University \\ Srabasti Chakravorty \\ Keele University \\ Paul Horrocks \\ Keele University School of Medicine \\ Catherine Jill Merrick ( $\nabla$ cjm48@cam.ac.uk) \\ University of Cambridge https://orcid.org/0000-0001-7583-2176
}

\section{Research}

Keywords: Plasmodium, var, antigenic variation, sirtuin, Sir2A, Sir2B, heat shock

Posted Date: April 28th, 2020

DOl: https://doi.org/10.21203/rs.3.rs-24665/v1

License: (9) This work is licensed under a Creative Commons Attribution 4.0 International License. Read Full License 


\section{Abstract}

Background: In the malaria parasite Plasmodium falciparum the expression of key 'var' virulence genes is regulated through epigenetic mechanisms. Two deacetylase enzymes of the sirtuin family have been implicated in this epigenetic control in laboratory-adapted parasites. A previous study of vargene expression in parasites isolated directly from Gambian malaria patients found that high expression levels of severe-disease-associated varvariants correlated with high expression of the PfSir $2 A$ sirtuin, and these expression patterns also correlated with patient phenotypes of fever and hyperlactataemia. Together, the observations suggest a mechanism through which stress phenotypes in the human host might be sensed via a parasite sirtuin, and virulence gene expression modulated accordingly.

Methods: In vitro experiments were conducted using recently-laboratory-adapted Kenyan isolates of $P$. falciparum to follow up the correlative findings of the field study. To investigate a potential cause-andeffect relationship between host stress factors and parasite gene expression, RT-PCR was used to measure the expression of sirtuins and vargenes after cultured parasites had been exposed to $2 \mathrm{~h}$ or $6 \mathrm{~h}$ of heat shock at $40^{\circ} \mathrm{C}$ or elevated lactate at $5 \mathrm{mM}$.

Results: Heat shock was shown to influence the expression of both sirtuins and vargenes, whereas exposure to lactate was not. Heat shock in the trophozoite stage resulted in modest upregulation of the expression of sirtuins, particularly $P f S i r 2 B$, by 2-3 fold in all strains tested. Interestingly, when heat shock was applied in ring stages $P f S i r 2 A$ was still upregulated but $P f S i r 2 B$ was downregulated. This correlated with a general upregulation of ring-stage vartranscription, and particularly of severe-disease-associated upsA and upsB vargenes, but there was no clear pattern in the dominant vargene(s) ultimately expressed by heat-shocked parasites.

Conclusions: This study demonstrates for the first time that heat stress in recently-laboratory-adapted patient isolates of $P$. falciparum results in altered sirtuin expression - PfSir2B as well as PfSir $2 A-$ and also the upregulation of var gene expression. These may be strategies evolved by the parasite to survive heat stress when a human host experiences malarial fevers. By contrast, the association between hyperlactataemia and sirtuin/vargene expression that was previously observed in vivo appears to be coincidental rather than causative.

\section{Background}

Currently, about 405,000 people die from malaria each year. This is almost always due to infection with the parasite species $P$. falciparum, which can cause severe and lethal malaria. Children make up $67 \%$ of this mortality [1]. Severe malaria manifests as three major syndromes: cerebral malaria, acute respiratory distress due to metabolic acidosis and severe anaemia (mainly in young children) [2]. Severe malaria is defined by a group of clinical factors including fever of above $39^{\circ} \mathrm{C}[3,4]$ and high blood lactate of $\geq$ $5 \mathrm{mM}[1,3,5,6]$. Fevers usually last between 2 and $6 \mathrm{~h}$ following a malaria paroxysm [7], while hyperlactataemia can persist for variable periods [3]. 
Severe malaria has been associated, in multiple studies, with parasites expressing particular members of the varvirulence gene family [8-14]. This is a large family of $\sim 60$ highly variable genes that all encode variants of the protein $P$. falciparum Erythrocyte Membrane Protein 1 (PfEMP1). PfEMP1 is a key virulence factor: it is exported to the surface of parasite-infected erythrocytes, where it mediates adhesion to host cell receptors found on the endothelial lining of blood vessels. Thus, infected erythrocytes can adhere in the microvasculature and avoid splenic clearance (reviewed in [15]). However, since the host immune system can recognise these PfEMP1 adhesins [16], the parasite has evolved to switch their expression regularly $[17,18]$ allowing immune evasion $[19]$ and thus persistent and repeated infections $[20,21]$.

Vargenes are grouped into the subgroups upsA, B, C and E based on sequence similarities in their upstream region, as well as their chromosomal location and direction of transcription [22]. There appears to be a 'hierarchy' of switching, in which genes of the upsA subgroup, encoding large and complex PfEMP1s that tend to correlate with severe disease [8-14], are expressed preferentially in immunologically naïve individuals [8]. There is some evidence that the upsB subgroup also associates with severe malaria, whereas the upsC group usually does not $[10,11]$.

Vargenes are generally considered to be expressed in a mutually exclusive fashion [18], although this may not be strictly true in all strains [23-25]. Mutually exclusive expression means that at any one time, the majority of the family is silenced epigenetically via heterochromatin formation [26]. The Plasmodium sirtuins, PfSir2A and B, contribute to this regulation of var expression by enforcing histone deacetylation and thus heterochromatic silencing. Disruption of either sirtuin gene in cultured 3D7 parasites leads to deregulated expression of many vargenes $[27,28]$.

These lines of evidence from in vitro cultured parasites informed a previous study of the correlation between sirtuin and vargene expression in in vivo human infections. RT-PCR was conducted on parasites directly isolated from Gambian patients with severe or mild malaria, revealing a correlation between elevated expression of PfSir2A and elevated expression of severe-disease-associated vargenes. This further correlated with patient phenotypes of fever and hyperlactataemia, leading to the hypothesis that these indictors of host stress might be detected by the parasite and translated, via sirtuin activity, into altered patterns of vargene expression [11]. A separate study subsequently conducted on East-African (Kenyan) rather than West-African (Gambian) isolates reproduced the correlation between fever, high PfSir2A expression, low pre-existing anti-PfEMP1 immunity, and - in this study - a generally elevated level of vartranscription rather than the elevation of any particular subgroup [29].

In this present study, conducted in vitro using recently-culture-adapted parasites, we endeavoured to establish whether the correlations detected in these field studies actually denote cause-and-effect relationships. P. falciparum parasites were accordingly subjected to high temperature and high lactate levels in vitro, mimicking the high body temperature and hyperlactatemia that occur in severe malaria.

\section{Methods}




\section{Parasite lines and culture}

The reference strain of $P$. falciparum, 3D7 (obtained from MR4), and 3 Kenyan strains (obtained from the European Malaria Reagent Repository, ID: ID9775, ID3518 and ID10668) were cultured in $0^{+}$erythrocytes obtained from the National Blood Transfusion Service (NBTS, Edgbaston, UK). Cultures were maintained at 4\% haematocrit in Roswell Park Memorial Institute (RPMI) 1640 medium containing $25 \mu \mathrm{g} / \mathrm{ml}$ gentamicin sulphate, $0.2 \%$ sodium bicarbonate (Sigma, USA), 0.2\% Albumax II (Life technologies, NewZealand), and $4.3 \%$ pooled human serum. Standard continuous culturing was carried out for 3D7 as described [30] or for the Kenyan strains, with more frequent media changes and at $1 \%$ haematocrit, as described [31]. Staging and parasitaemia were assessed from a thin blood smear stained with Hemacolor ${ }^{\circledR}$ Rapid (VWR, UK). Storage and disposal of blood were in accordance with the Human Tissue Authority (HTA) license held by Keele University.

\section{Cloning and expression of luciferase reporter gene}

The firefly luciferase gene (F/uc) was amplified from an existing expression cassette [32] using primers P1 and P2 (Additional file 1, Table S1), and ligated into the pLNSir2GFP plasmid [11] between the Afll and Avrl sites. Cloning was conducted in the PMC103 strain of E. coli (ATTC). The resulting plasmid, pLNSir2aproluc (Additional file 2, Figure S1A), was transfected into the 3D7 strain as previously described [33].

\section{Synchronization of parasites}

Double sorbitol synchronization was carried out as described [34], either $16 \mathrm{~h}$ apart (for the experiments with trophozoites) or $20 \mathrm{~h}$ apart (for the experiments with rings).

\section{Stress treatment of parasites}

Trophozoites were subjected to high temperature $\left(40{ }^{\circ} \mathrm{C}\right)$ and/or to $5 \mathrm{mM}$ sodium lactate for 2 or $6 \mathrm{~h}$. The rings were exposed to these stressors at $2 \pm 2$ hours post invasion (hpi) for $6 \mathrm{~h}$ only. This level of heat shock was chosen because temperatures above $40{ }^{\circ} \mathrm{C}$ were previously shown to kill most mature forms of the asexual stage [35]. Control samples, cultured under normal culture conditions, were included for all timepoints. After the stress, the medium was completely changed and the cultures were used for transcriptional analysis: RNA was extracted immediately, or after $10 \mathrm{~h}$ recovery (ring-stage experiments only). An aliquot of each culture was also diluted to parasitaemia of $0.5 \%$ (for trophozoites) or $0.1 \%$ (for rings) with $4 \%$ haematocrit in complete media. This was then cultured for $48 \mathrm{~h}$ (for trophozoites) or $72 \mathrm{~h}$ (for rings) to determine survival rate. For this, parasite counting by microscopy was conducted against up to 3000 erythrocytes and SYBR Green 1-based fluorescence assessment was conducted as described below. Survival was calculated using the equation: $(\mathrm{gt} / \mathrm{gc}) \times 100$, where $\mathrm{gt}=$ parasitaemia or fluorescence of the test sample and gc = parasitaemia or fluorescence of the control sample.

\section{SYBR Green 1-based fluorescence assessment}


Growth was assessed using the SYBR Green 1-based fluorescence method by mixing $100 \mu \mathrm{l}$ of the parasite cultures with $100 \mu \mathrm{l}$ of lysis buffer containing SYBR green at a 1:5000 dilution (lysis buffer = $20 \mathrm{mM}$ Tris $\mathrm{pH} 7.5,5 \mathrm{mM}$ EDTA, $0.008 \% \mathrm{v} / \mathrm{v}$ saponin and $0.08 \% \mathrm{v} / \mathrm{v}$ Triton X-100) in the wells of a 96 -well black plate (Cellstar, Greiner-bio-one, Germany) in the dark. The plates were then incubated for $1 \mathrm{~h}$ in the dark and the fluorescence intensity was measured using $490 \mathrm{~nm}$ excitation and 510-570 nm emission filter in a Glomax-Multi Detection System (Promega, UK). Raw fluorescence values were used to determine statistical significance.

\section{Luciferase reporter assay}

Stress experiments were conducted in the 3D7 PfSir2A-luc reporter line as above, using trophozoite stages only. A previously characterised luciferase reporter line, Dd2 Pfpcna-luc [32] was also included as a control in these experiments. Bioluminiscence of the transgenic lines was measured immediately after stress using the improved Single-Step Lysis Protocol [36] in a Glomax-Multi Detection System (Promega, UK). Measurements were converted to relative luciferase activity as compared to the untreated transgenic line.

\section{Gene expression analysis}

Total RNA was extracted from each sample culture as described previously 546 Kyes,Sue 2000, but rather than adding Trizol to the iRBCs, Trizol was added directly to the parasite pellet after release from RBCs by saponin lysis and washing with PBS. RNA yield was quantified using a Nanospec 1000 (Thermoscientific, USA). Contaminating DNA was removed using the wipe out buffer in the QuantiTect ${ }^{\circledR}$ Reverse Transcription Kit (QIAGEN GmbH, Germany). cDNA was synthesized with this kit and the absence of contaminating genomic DNA was verified by PCR across the intron of the gene PF3D7_0424300, as described previously [37]. Relative gene expression of the sirtuins and var genes was determined by RTqPCR in a StepOne Plus ${ }^{\text {TM }}$ Real-time PCR machine (Thermo Fischer Scientific) using SensiFAST ${ }^{\text {TM }}$ SYBR (Bioline, UK), cDNA (diluted at most 1:20) and primers $(0.125 \mu \mathrm{M})$ in a $20 \mu \mathrm{l}$ reaction volume. Cycling conditions were $50^{\circ} \mathrm{C}$ for $2 \mathrm{~min}$, initial denaturation at $95^{\circ} \mathrm{C}$ for $3 \mathrm{~min}$, followed by 40 cycles of denaturation at $95^{\circ} \mathrm{C}$ for $15 \mathrm{~s}$, annealing at $54^{\circ} \mathrm{C}$ for $40 \mathrm{~s}$ and elongation at $60{ }^{\circ} \mathrm{C}$ for 1 min. A melt-curve step was included to verify the specificity of the reactions, in addition to agarose gel electrophoresis, if required. Reference genes used as controls were PF3D7_0717700 (serine tRNA-ligase), PF3D7_1444800 (FBP aldolase), PF3D7_1246200 (actin), PF3D7_0501300(SBP1) andPF3D7_1370300 (MAHRP1), as previously described [11]. Primers to the target genes PfSir2A, PfSir2B [11], the major vargene groupings [10], and the conserved acidic terminal segment (ATS) of all vargenes [11] were used as published. Primers to the PfHsp70 (P3 and P4) and MAHRP1 (P5 and P6) were designed using Snapgene software and their sequences are in Additional file 1, Table S1.

Relative copy number (RCN) of each target gene was calculated via $2^{-\Delta \Delta C t}$ (Livak, Schmittgen 2001), normalized to the average of 3 reference genes (serine-tRNA ligase, FBP aldolase and actin) for the sirtuin analysis; or to the average of all 5 reference genes for vargene group analysis. Relative copy numbers for each gene were then compared to gene expression in unstressed control parasites using the $\Delta \Delta \mathrm{Cq}$ 
method, to obtain the relative quantity $\left(R Q=2^{-\Delta \Delta C q}\right)$ in technical triplicates. Experiments with the laboratory strain, 3D7 were done in independent biological triplicates, while those with the field strains were done in independent duplicates. RCNs $\left(2^{-\Delta \mathrm{Cq}}\right)$ were used to determine statistical significance.

\section{Statistical analysis}

Raw data were tested for normality, using the Kolmogorov-Smirnov test for normality, and the majority of the datasets did not follow a normal distribution. Statistical significance was therefore determined by using the Kruskal-Wallis test with Dunn's post-test, using GraphPad Prism version 5.01 for Windows (GraphPad Software, San Diego California USA). A Kruskal-Wallis test $p$ value of less than 0.05 indicated that one or more of the treatments caused statistically significant changes.

\section{Results}

\section{Heat shock adversely affects parasite survival whereas lactate exposure does not}

In order to choose appropriate parameters for exposing parasites to either heat shock or elevated lactate, we first measured the parasite survival following such exposures, both at the ring stage and at the trophozoite stage. Trophozoites are known to be more sensitive to heat shock than rings [38], and although comparable stage-specific data are not published for lactate sensitivity, the trophozoite is also the most metabolically active stage, at which lactate production is highest. Cultures were treated at either $2 \pm 2$ hours post-invasion (hpi), or at $28 \pm 4 \mathrm{hpi}$ (primarily young rings and young trophozoites respectively). Exposure to stress was for $2 \mathrm{~h}$ or $6 \mathrm{~h}$ and consisted of heat shock at $40^{\circ} \mathrm{C}$ or $5 \mathrm{mM}$ added lactate. These were chosen to mimic, respectively, common levels of fever in the human host and the WHO threshold for hyperlactataemia that defines severe malaria [2]. Survival was measured $48 \mathrm{~h}$ after treatment of the trophozoites or $72 \mathrm{~h}$ after treatment of the rings, thus allowing reinvaded rings to develop into trophozoites, when parasitaemia can be easily assessed. Four parasite strains were used: the reference strain 3D7 and three recently laboratory-adapted strains derived from Kenyan malaria patients.

In trophozoites, heat shock resulted in $0-20 \%$ death following a $2 \mathrm{~h}$ exposure and $20-40 \%$ death following a $6 \mathrm{~h}$ exposure (Fig. 1A). The extent of heat-shock-induced death was strain-dependent, with one Kenyan strain (ID9775) being markedly more sensitive than the other two, and 3D7 being of intermediate sensitivity. Interestingly, we observed that the most heat-sensitive field strain (ID9775) was the one that grew most rapidly and robustly in normal culture conditions. These results, measured via the SYBR Green-1 Fluorescence method, which measures levels of parasite DNA, were broadly corroborated by microscopy, conducted on two of the four strains as an independent measure of viable parasite numbers (Fig. 1B). Microscopy tended to yield a lower survival rate, because the SYBR Green-1 Fluorescence method also measures background fluorescence from potentially 'dead' parasite DNA, whereas microscopy allows live and dead parasites to be distinguished. By contrast to heat shock, lactate 
exposure did not have a significant adverse effect on parasite survival (Fig. 1A, B) and when both stressors were applied together, results were very similar to those seen after heat shock alone (Fig. 1A, B).

When heat shock was applied to ring-stage parasites, survival after $72 \mathrm{~h}$ was more variable between strains than it had been at the trophozoite stage (Fig. 1C, D). Kenyan strain ID3518 was almost completely insensitive, whereas rings of strains ID 9775 and ID 10668 were significantly affected (27\% and $38 \%$ death, respectively, i.e. almost as severely affected as their trophozoites). As before, microscopy yielded a more severe assessment of heat-induced death than the SYBR Green-1 Fluorescence assessment (Fig. 1C cf Fig. 1D). Lactate exposure for $6 \mathrm{~h}$ at the ring stage again had little effect on parasite survival (Fig. 1C, D).

\section{Heat shock modulates the expression of sirtuins in P. falciparum trophozoites}

Having established heat shock conditions that caused only a moderate amount of parasite death in both trophozoites and rings, we measured the expression of sirtuin genes immediately after heat shock, as well as the expression of the gene encoding heat shock protein 70 ( $h s p 70)$ as a positive control. These RT-PCR experiments were conducted first on trophozoites: the stage that was previously reported to upregulate PfSir2A expression after heat shock [35]. Hsp70 expression was indeed upregulated, 3-7 fold in most strains, with considerable strain-to-strain variation $(p<0.0001, n=27)($ Fig. $2 A)$. This response was heat-shock-specific because $h s p 70$ was not upregulated after lactate exposure, as expected.

PfSir2A expression trended upwards in most strains after heat shock, particularly at the $2 \mathrm{~h}$ time point and somewhat less so at the $6 \mathrm{~h}$ time point. However, upregulation was usually less than 2 -fold and did not reach statistical significance (Fig. 2B). PfSir2B expression, meanwhile, also trended upwards, generally by 2-3-fold, and reached statistical significance in several strains $(p=0.0031, n=27$ at $2 h ; p=$ $0.0495, n=27$ at 6 h) (Fig. 2C). There was no significant difference in the response to heat shock alone versus heat shock combined with $5 \mathrm{mM}$ lactate, thus suggesting that heat shock was the main factor modulating transcription of the genes measured here.

Since the Sir2A gene appeared only modestly upregulated after heat shock, we designed a second independent approach to measure this response. A luciferase reporter gene was cloned under the presumptive Sir2A promoter ( $1.7 \mathrm{~kb}$ of the gene's upstream sequence) and the reporter gene was transfected into 3D7 parasites, where it was shown to follow an expression profile similar to that of the endogenous Sir2a gene across the intraerythrocytic cycle [39](Additional file 2, Figure S1B). Unfortunately, although this system may be of use in other applications, it proved unsuitable for heat shock experiments because the luciferase was severely destabilised at $40{ }^{\circ} \mathrm{C}$ : activity dropped by $\sim 80 \%$ after a $2 \mathrm{~h}$ heat shock, irrespective of the promoter driving luciferase expression (Additional file 2, Figure S1C), thus obscuring any promoter-mediated regulation at the transcriptional level.

Heat shock modulates the expression of sirtuins and var genes in P. falciparum ring stages, whereas lactate does not 
Figure 2 shows that exposure to heat shock can modulate the expression of both parasite sirtuins, particularly PfSir2B, at least in trophozoite-stage parasites. We therefore proceeded to investigate the same responses in ring-stage parasites, i.e. the stage at which vargenes are also expressed. Sirtuin and vargene expression were measured immediately after the rings had been exposed to heat shock, elevated lactate, or both stressors combined for $6 \mathrm{~h}$, i.e. expression was measured at $\sim 8 \mathrm{hpi}$. The same genes were also measured $10 \mathrm{~h}$ later, at $\sim 18 \mathrm{hpi}-$ around the time of maximum expression for active var gene(s) [40]. Kenyan field strains (ID9775 and ID3518) were selected for these experiments and, since var gene families are hyper-diverse, var expression was measured using general primer sets that were previously developed to detect conserved regions within each ups-group of var genes [10]. These same primers were used in the study of Gambian field strains which originally reported the association between vargene expression, sirtuin expression, patient fever and hyperlactataemia [11].

Exposure of ring-stage parasites to heat shock induced the upregulation of $h s p 70$ (Fig. 3A), as was previously seen in trophozoites (Fig. 2A). Upregulation was at most 2.5-fold and the magnitude of the response varied between strains, with strain ID9775 appearing almost entirely refractory at the ring stage, despite being clearly responsive at the trophozoite stage. PfSir2A expression trended upwards in both strains immediately after heat shock, similar to the response in trophozoites, but the increase was again less than 2-fold and did not reach statistical significance. Nevertheless, after $10 \mathrm{~h}$ of recovery at $37^{\circ} \mathrm{C}$, PfSir2A expression remained elevated, reaching statistical significance in strain ID3518 (Fig. 3B). PfSir2B, meanwhile, showed a response that contrasted with its response in trophozoites: immediately after heat shock, PfSir2B expression was downregulated by 2-2.1-fold, but returned to baseline levels following $10 \mathrm{~h}$ of recovery (Fig. 3C).

In concert with these changes in sirtuin expression, changes in var gene expression after heat shock were also measured (Fig. 3D, E). Immediately after heat shock (i.e. $8 \mathrm{hpi}$ ), both strains showed a general upregulation of vartranscription: 1.5-2-fold upregulation was measured with the pan-var 'ATS' primer (which detects the conserved sequence encoding the 'Acidic Terminal Segment' of PfEMP1), although this reached statistical significance only in strain ID9775. Primers specific to the various ups groups A-E suggested that most groups, except for upsE, contributed to this, with subtelomeric upsA and upsB genes contributing particularly strongly in strain ID3518 (Fig. 3D). Interestingly, $10 \mathrm{~h}$ later at $18 \mathrm{hpi}$ the overall level of vartranscript remained elevated, by $\sim 4$-fold in the more strongly responding ID3518 strain and 1.5 -fold in strain ID9775. However, the most strongly upregulated vargene had changed in both strains. A upsC gene was elevated in strain ID3518, while strain ID9775 had upregulated the upsE gene var2csa (Fig. 3E).

Having established that heat stress could indeed induce changes in both sirtuin and vargene expression when applied to ring-stage parasites, we proceeded to compare this with the transcriptional response to lactate exposure. This caused no consistent change in sirtuin expression (Fig. 4A, B), as had previously been shown in trophozoites, and there was also no clear or consistent change in var gene expression at the peak $18 \mathrm{hpi}$ timepoint. When var expression was measured at $8 \mathrm{hpi}$, immediately after lactate exposure, one of the two strains did show a marked upregulation in groups B and C2 vartranscripts 
(Fig. 4C). This, however, was not sustained by $18 \mathrm{hpi}$ (Fig. 4D), casting some doubt upon its potential phenotypic significance.

Finally, we tested the transcriptional effect upon ring-stage parasites of combining heat shock with lactate exposure. In trophozoites, this had yielded similar results - in terms of both parasite survival and hsp70/sirtuin expression - as had heat shock alone. The same was evidently true in ring-stage parasites: hsp70, PfSir2A and PfSir2B expression all responded almost identically regardless of the presence or absence of $5 \mathrm{mM}$ lactate (compare Fig. 5A-C with 3A-C). Var expression responses were likewise broadly similar (Fig. 5D, E), particularly at $18 \mathrm{hpi}$. There was an overall upregulation in transcript levels, stronger in strain ID3518 than ID9775, and the predominantly upregulated genes at $18 \mathrm{hpi}$ were a upsC gene in ID3518 and the upsE gene in ID9775. Subtle differences were seen immediately after treatment at $8 \mathrm{hpi}$, with the overall upregulation being less severe in this dataset, and the ID3518 strain apparently already showing strong upregulation of the eventually-dominant upsC gene. However, the biological outcome in terms of expressed PfEMP1 would probably be very similar in both datasets.

\section{Discussion}

This is the first study to use recently laboratory-adapted field strains, rather than just the established laboratory parasite 3D7, to examine links between $P$. falciparum sirtuin expression and elevated temperature. It is also the first to report a link between PfSir2B, as well as PfSir2A, and fever temperatures.

High body temperature and high blood lactate were previously shown to be associated with upregulated sirtuin and varexpression in Gambian field isolates of $P$. falciparum causing severe disease [11]. In a separate study, fever alone was associated with increased expression of PfSir $2 A$ and vargenes in Kenyan field isolates [29]. However, cause-and effect relationships were not established in these studies. Here we have shown that trophozoites of $P$. falciparum consistently increased their expression of both sirtuins after heat shock, with $P f S i r 2 B$ responding more strongly than $P f S i r 2 A$, whereas ring stages responded to heat shock with a transient downregulation of $P f S i r 2 B$ and upregulation of PfSir2A. Our results are partially consistent with a previous in vitro study of the heat shock response in 3D7 [35], which found PfSir2A to be upregulated by 2.4 -fold after a $41{ }^{\circ} \mathrm{C}$ heat shock. PfSir2B, however, did not reach a 2 -fold threshold, probably because asynchronous cultures were used, masking the stage-specific changes that we observed here in PfSir2B.

These changes in sirtuin expression correlated with increased expression of vargenes, consistent with the published findings from direct patient isolates $[11,29]$ and from in vitro heat-shocked 3D7 parasites [35]. In fact, the levels of var gene upregulation were higher in this study than reported previously [35], possibly because heat shock was applied for longer ( $6 \mathrm{~h}$ vs $2 \mathrm{~h}$ ) or because our field strains were more responsive than the 3D7 laboratory strain. Furthermore, there was predominant expression of severedisease-associated vargenes in early rings immediately after heat shock: a novel finding in this study, and one that might reflect heat-induced changes to the structure of subtelomeric chromatin where upsA and upsB vargenes are encoded. This, however, was not sustained at $18 \mathrm{hpi}$, by which time overall 
transcript levels remained elevated but a different vargene was predominantly upregulated in both strains (upsC and upsE).

There is conflicting literature on the subject of whether elevated var expression actually leads to elevated expression of PfEMP1 on the surface of the infected RBC $[35,41]$ and this question was not re-addressed in our present study. However, if it does then this could be a mechanism enabling parasites to cytoadhere more efficiently and thus survive better following an immune-response-induced fever. A second theory has also been published, in which $P$. falciparum immune evasion during chronic infections involves low vargene expression [29]. It may be that this indeed occurs in semi-immune individuals, who are less likely to experience symptomatic fevers, whereas parasites in symptomatic, minimally-immune individuals may tend to respond differently, by increasing var expression and cytoadherence. Finally, during the preparation of this manuscript, a third study was published examining var and PfSir2A expression in parasite-positive individuals in Mozambique [42]. Unlike the Gambian and Kenyan patients, these subjects were identified via a community survey, with none being severely ill or attending a clinic, although some did have an actual or reportedly-recent fever. PfSir2A transcription was actually lower in the febrile group than in the afebrile group - perhaps suggesting that our hypothesis concerning PfSir $2 A$ levels is only significant in cases of more severe and symptomatic disease.

By contrast with the results discussed above concerning the relationship between fever and virulence gene expression, we did not detect any comparable transcriptional response to elevated lactate levels. This suggests that although hyperlactataemia was clearly identified in our original field study as a correlate of sirtuin upregulation [11], the correlation is probably not causative. Alternatively, the level of lactate used here may not have been high enough to see an effect $-5 \mathrm{mM}$ is at, not above, the clinical threshold. However, only $26 \%$ of the original patient cohort in the Gambian study [11] had lactate levels > $5 \mathrm{mM}$, suggesting that in vivo this is indeed relatively rare, and aberrantly high.

\section{Conclusion}

Overall, the data presented here suggest that observations made in both East- and West-African field studies of patients with severe versus mild malaria $[11,29]$ do reflect a causative relationship between fever in the human host and expression of varvirulence genes. Our study does not actually prove that the change is mediated via sirtuin activity, but it does correlate with changes in sirtuin transcription (further proof would require experiments, such as vargene ChIP for modified histones, or generation of sirtuin knockouts, which would be challenging in newly-cultured field strains). Nevertheless, the idea is consistent with some existing data from the 3D7 strain showing that PfSir2A overexpression can correlate with vargene upregulation [11]. The interplay between PfSir2A and PfSir2B remains uncharacterised and is probably complex. However, the upregulation versus down-regulation of the two sirtuins that we observed when ring stages experienced heat shock may point to opposing roles for these two enzymes - a potentially interesting area for future study.

\section{Declarations}


Ethics approval and consent to participate

Not applicable. All parasite strains were obtained from registered repositories, rather than directly from human patients. De-identified human erythrocytes in which $P$. falciparum strains were cultured was obtained from the National Blood Service. The use of blood is regulated under the Human Tissues Act 2004 and Keele University is an approved user of the National Blood Service.

\section{Consent for publication}

Not applicable

\section{Availability of data and material}

All data generated or analysed during this study are included in this published article.

\section{Competing interests}

The authors declare that they have no competing interests.

\section{Funding}

This work was partially funded by the tertiary Education Trust Fund (TETfund) Nigeria. The funders had no role in the study design, data collection and analysis, decision to publish, or preparation of the manuscript.

\section{Authors' contributions}

LOA conducted experiments, designed some aspects of the study, analysed data with the assistance of DRH and drafted the manuscript. PDH assisted in the design of the luciferase experiments, provided the control luciferase-expressing strain and reviewed the manuscript. CJM designed the study, supervised the study with the assistance of SJC, and wrote the manuscript. All authors read and approved the final manuscript.

\section{Acknowledgements}

We are grateful to Dr. Imran Ullah for help in developing the heat shock experiments.

\section{Abbreviations}

DNA - Deoxyribonucleic acid

EDTA - Ethylenediaminetetraacetic acid Hsp70 - Heat shock protein-70

MR4 - The Malaria Research and Reference Reagent Resource Center

MSF - Malaria SYBR Green 1-based fluorescence assay 
PfEMP1 - P. falciparum Erythrocyte Membrane Protein 1

PBS - Phosphate-buffered saline

PfSir2A - Plasmodium falciparum silent information regulator 2A

PfSir2B - Plasmodium falciparum silent information regulator 2B

RBC - Red blood cell

RNA - Ribonucleic acid

RT-PCR/RT-qPCR - Quantitative reverse transcription polymerase chain reaction

WHO - World health organization

\section{References}

1. WHO: World Malaria Report 2019. 2019.

2. WHO: Severe Malaria.Tropical Medicine and International Health 2014, 19:7-131.

3. Krishna S, Waller DW, ter Kuile F, Kwiatkowski D, Crawley J, Craddock CF, Nosten F, Chapman D, Brewster D, Holloway PA, et al.: Lactic acidosis and hypoglycaemia in children with severe malaria: pathophysiological and prognostic significance.Trans R Soc Trop Med Hyg 1994, 88:67-73.

4. Mockenhaupt FP, Ehrhardt S, Burkhardt J, Bosomtwe SY, Laryea S, Anemana SD, Otchwemah RN, Cramer JP, Dietz E, Gellert S, Bienzle U: Manifestation and outcome of severe malaria in children in northern Ghana.Am J Trop Med Hyg 2004, 71:167-172.

5. Agbenyega T, Angus BJ, Bedu-Addo G, Baffoe-Bonnie B, Guyton T, Stacpoole PW, Krishna S: Glucose and lactate kinetics in children with severe malaria.J Clin Endocrinol Metab 2000, 85:1569-1576.

6. Casals-Pascual C, Kai O, Lowe B, English M, Williams TN, Maitland K, Newton CR, Peshu N, Roberts DJ: Lactate levels in severe malarial anaemia are associated with haemozoin-containing neutrophils and low levels of IL-12.Malar J 2006, 5:101.

7. Bartoloni A, Zammarchi L: Clinical aspects of uncomplicated and severe malaria.Mediterr J Hematol Infect Dis 2012, 4:e2012026.

8. Jensen AT, Magistrado P, Sharp S, Joergensen L, Lavstsen T, Chiucchiuini A, Salanti A, Vestergaard LS, Lusingu JP, Hermsen R, et al: Plasmodium falciparum associated with severe childhood malaria preferentially expresses PfEMP1 encoded by group A var genes.J Exp Med 2004, 199:1179-1190.

9. Kyriacou HM, Stone GN, Challis RJ, Raza A, Lyke KE, Thera MA, Kone AK, Doumbo OK, Plowe CV, Rowe JA: Differential var gene transcription in Plasmodium falciparum isolates from patients with cerebral malaria compared to hyperparasitaemia.Mol Biochem Parasitol 2006, 150:211-218.

10. Rottmann M, Lavstsen T, Mugasa JP, Kaestli M, Jensen AT, Muller D, Theander T, Beck HP: Differential expression of var gene groups is associated with morbidity caused by Plasmodium 
falciparum infection in Tanzanian children.Infect Immun 2006, 74:3904-3911.

11. Merrick CJ, Huttenhower C, Buckee C, Amambua-Ngwa A, Gomez-Escobar N, Walther M, Conway DJ, Duraisingh MT: Epigenetic dysregulation of virulence gene expression in severe Plasmodium falciparum malaria.J Infect Dis 2012, 205:1593-1600.

12. Abdi Al, Fegan G, Muthui M, Kiragu E, Musyoki JN, Opiyo M, Marsh K, Warimwe GM, Bull PC: Plasmodium falciparum antigenic variation: relationships between widespread endothelial activation, parasite PfEMP1 expression and severe malaria.BMC Infect Dis 2014, 14:170.

13. Storm J, Jespersen JS, Seydel KB, Szestak T, Mbewe M, Chisala NV, Phula P, Wang CW, Taylor TE, Moxon CA, et al: Cerebral malaria is associated with differential cytoadherence to brain endothelial cells.EMBO Mol Med 2019, 11.

14. Claessens A, Adams Y, Ghumra A, Lindergard G, Buchan CC, Andisi C, Bull PC, Mok S, Gupta AP, Wang $\mathrm{CW}$, et al: A subset of group A-like var genes encodes the malaria parasite ligands for binding to human brain endothelial cells. Proc Natl Acad Sci U S A 2012, 109:E1772-1781.

15. Pasternak ND, Dzikowski R: PfEMP1: an antigen that plays a key role in the pathogenicity and immune evasion of the malaria parasite Plasmodium falciparum. Int J Biochem Cell Bio/ 2009, 41:1463-1466.

16. Newbold Cl, Pinches R, Roberts DJ, Marsh K: Plasmodium falciparum: the human agglutinating antibody response to the infected red cell surface is predominantly variant specific.Exp Parasitol 1992, 75:281-292.

17. Roberts DJ, Craig AG, Berendt AR, Pinches R, Nash G, Marsh K, Newbold Cl: Rapid switching to multiple antigenic and adhesive phenotypes in malaria.Nature 1992, 357:689-692.

18. Scherf A, Hernandez-Rivas R, Buffet P, Bottius E, Benatar C, Pouvelle B, Gysin J, Lanzer M: Antigenic variation in malaria: in situ switching, relaxed and mutually exclusive transcription of var genes during intra-erythrocytic development in Plasmodium falciparum.Embo J 1998, 17:5418-5426.

19. Abdi Al, Hodgson SH, Muthui MK, Kivisi CA, Kamuyu G, Kimani D, Hoffman SL, Juma E, Ogutu B, Draper SJ, et al: Plasmodium falciparum malaria parasite var gene expression is modified by host antibodies: longitudinal evidence from controlled infections of Kenyan adults with varying natural exposure.BMC Infect Dis 2017, 17:585.

20. Franks S, Koram KA, Wagner GE, Tetteh K, McGuinness D, Wheeler JG, Nkrumah F, Ranford-Cartwright L, Riley EM: Frequent and persistent, asymptomatic Plasmodium falciparum infections in African infants, characterized by multilocus genotyping.J Infect Dis 2001, 183:796-804.

21. Ashley EA, White NJ: The duration of Plasmodium falciparum infections.Malar J 2014, 13:500.

22. Lavstsen T, Salanti A, Jensen AT, Arnot DE, Theander TG: Sub-grouping of Plasmodium falciparum 3D7 var genes based on sequence analysis of coding and non-coding regions.Malar J 2003, 2:27.

23. Joergensen L, Bengtsson DC, Bengtsson A, Ronander E, Berger SS, Turner L, Dalgaard MB, Cham GK, Victor ME, Lavstsen T, et al: Surface co-expression of two different PfEMP1 antigens on single plasmodium falciparum-infected erythrocytes facilitates binding to ICAM1 and PECAM1.PLOS Pathog, 6:e1001083. 
24. Merrick CJ, Jiang RH, Skillman KM, Samarakoon U, Moore RM, Dzikowski R, Ferdig MT, Duraisingh MT: Functional analysis of sirtuin genes in multiple Plasmodium falciparum strains.PLoS One 2015, 10:e0118865.

25. Bachmann A, Bruske E, Krumkamp R, Turner L, Wichers JS, Petter M, Held J, Duffy MF, Sim BKL, Hoffman SL, et al: Controlled human malaria infection with Plasmodium falciparum demonstrates impact of naturally acquired immunity on virulence gene expression.PLoS Pathog 2019, 15:e1007906.

26. Freitas-Junior LH, Hernandez-Rivas R, Ralph SA, Montiel-Condado D, Ruvalcaba-Salazar OK, RojasMeza AP, Mancio-Silva L, Leal-Silvestre RJ, Gontijo AM, Shorte S, Scherf A: Telomeric heterochromatin propagation and histone acetylation control mutually exclusive expression of antigenic variation genes in malaria parasites.Cel/ 2005, 121:25-36.

27. Duraisingh MT, Voss TS, Marty AJ, Duffy MF, Good RT, Thompson JK, Freitas-Junior LH, Scherf A, Crabb BS, Cowman AF: Heterochromatin silencing and locus repositioning linked to regulation of virulence genes in Plasmodium falciparum.Cel/ 2005, 121:13-24.

28. Tonkin CJ, Carret CK, Duraisingh MT, Voss TS, Ralph SA, Hommel M, Duffy MF, Silva LM, Scherf A, Ivens $A$, et al: Sir2 paralogues cooperate to regulate virulence genes and antigenic variation in Plasmodium falciparum.PLoS Biol 2009, 7:e84.

29. Abdi Al, Warimwe GM, Muthui MK, Kivisi CA, Kiragu EW, Fegan GW, Bull PC: Global selection of Plasmodium falciparum virulence antigen expression by host antibodies.Sci Rep 2016, 6:19882.

30. Ménard RE: Malaria: Methods and Protocols.2013.

31. Radfar A, Mendez D, Moneriz C, Linares M, Marin-Garcia P, Puyet A, Diez A, Bautista JM: Synchronous culture of Plasmodium falciparum at high parasitemia levels.Nat Protoc 2009, 4:1899-1915.

32. Wong EH, Hasenkamp S, Horrocks P: Analysis of the molecular mechanisms governing the stagespecific expression of a prototypical housekeeping gene during intraerythrocytic development of P. falciparum.J Mol Biol 2011, 408:205-221.

33. Deitsch K, Driskill C, Wellems T: Transformation of malaria parasites by the spontaneous uptake and expression of DNA from human erythrocytes.Nucleic Acids Res 2001, 29:850-853.

34. Lambros C, Vanderberg JP: Synchronization of Plasmodium falciparum erythrocytic stages in culture.J Parasitol 1979, 65:418-420.

35. Oakley MS, Kumar S, Anantharaman V, Zheng H, Mahajan B, Haynes JD, Moch JK, Fairhurst R, McCutchan TF, Aravind L: Molecular factors and biochemical pathways induced by febrile temperature in intraerythrocytic Plasmodium falciparum parasites.Infect Immun 2007, 75:20122025.

36. Hasenkamp S, Wong EH, Horrocks P: An improved single-step lysis protocol to measure luciferase bioluminescence in Plasmodium falciparum.Malar J 2012, 11:42.

37. Merrick CJ, Dzikowski R, Imamura H, Chuang J, Deitsch K, Duraisingh MT: The effect of Plasmodium falciparum Sir2a histone deacetylase on clonal and longitudinal variation in expression of the var family of virulence genes.Int J Parasitol 2010, 40:35-43. 
38. Kwiatkowski D: Febrile temperatures can synchronize the growth of Plasmodium falciparum in vitro.J Exp Med 1989, 169:357-361.

39. Otto TD, Wilinski D, Assefa S, Keane TM, Sarry LR, Bohme U, Lemieux J, Barrell B, Pain A, Berriman M, et al: New insights into the blood-stage transcriptome of Plasmodium falciparum using RNA-Seq. $\mathrm{Mol}$ Microbio/ 2010, 76:12-24.

40. Dahlback M, Lavstsen T, Salanti A, Hviid L, Arnot DE, Theander TG, Nielsen MA: Changes in var gene mRNA levels during erythrocytic development in two phenotypically distinct Plasmodium falciparum parasites.Malar J 2007, 6:78.

41. Udomsangpetch R, Pipitaporn B, Silamut K, Pinches R, Kyes S, Looareesuwan S, Newbold C, White $\mathrm{NJ}$ : Febrile temperatures induce cytoadherence of ring-stage Plasmodium falciparum-infected erythrocytes.Proc Natl Acad Sci U S A 2002, 99:11825-11829.

42. Gupta H, Galatas B, Matambisso G, Nhamussua L, Cistero P, Bassat Q, Casellas A, Macete E, Aponte $\mathrm{JJ}$, Sacoor C, et al: Differential expression of var subgroups and PfSir2a genes in afebrile Plasmodium falciparum malaria: a matched case-control study.Malar J 2019, 18:326.

\section{Figures}




\section{A}

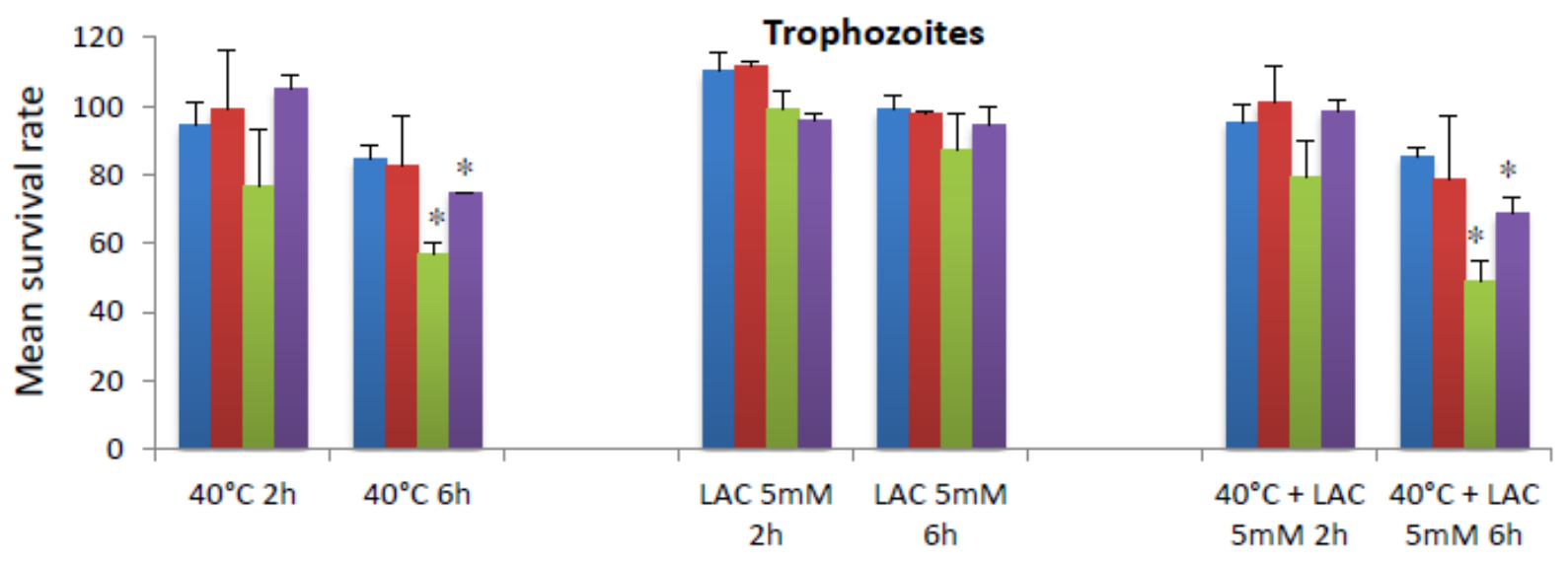

B

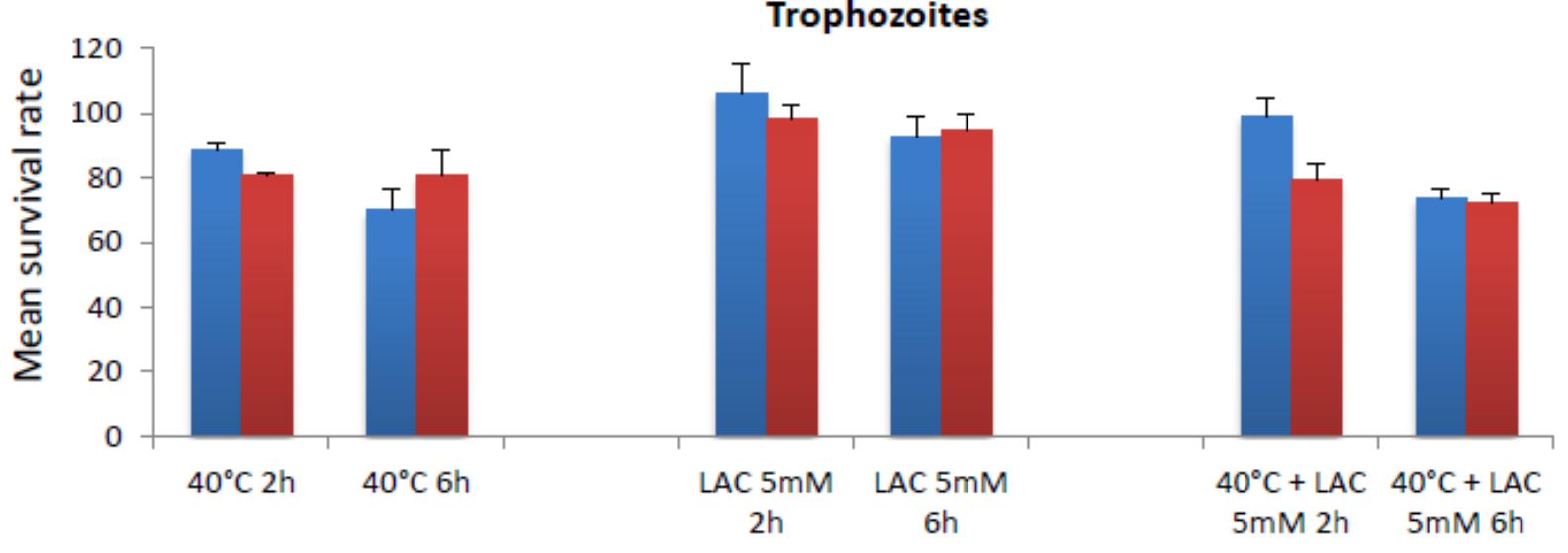

C

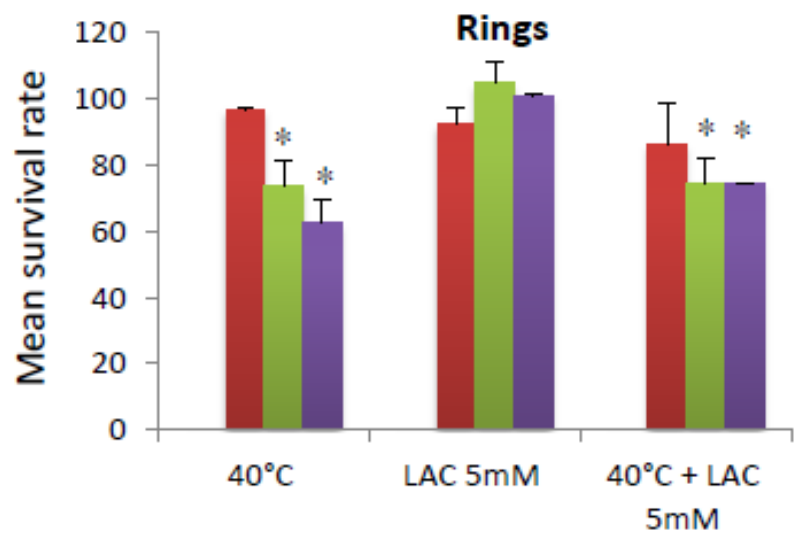

D

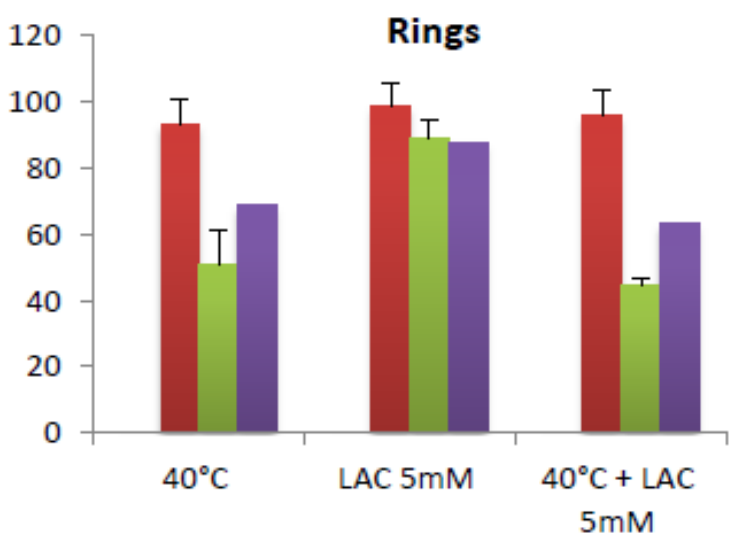

\section{Figure 1}

Post-stress survival of P. falciparum after exposure to lactate and heat shock. A. Parasite survival $48 \mathrm{~h}$ after stress treatment for $2 \mathrm{~h}$ or $6 \mathrm{~h}$ at the trophozoite stage, relative to growth of a control culture, measured via DNA content in a SYBR-Green-1 based assay. B. Parasite survival as in a, assessed by microscopy. C. Parasite survival $72 \mathrm{~h}$ after stress treatment for $6 \mathrm{~h}$ at the ring stage, relative to growth of a control culture, measured via DNA content in a SYBR-Green-1 based assay. D. Parasite survival as in C., 
assessed by microscopy. Blue bars, 3D7; red bars, ID3518; green bars, ID9775; purple bars, ID10668. Mean survival rates are from biological duplicates for the African strains, or triplicates for 3D7. Error bars represent standard error. *, $p<0.05$, Kruskal-Wallis test with Dunn's post test of raw fluorescence reads.
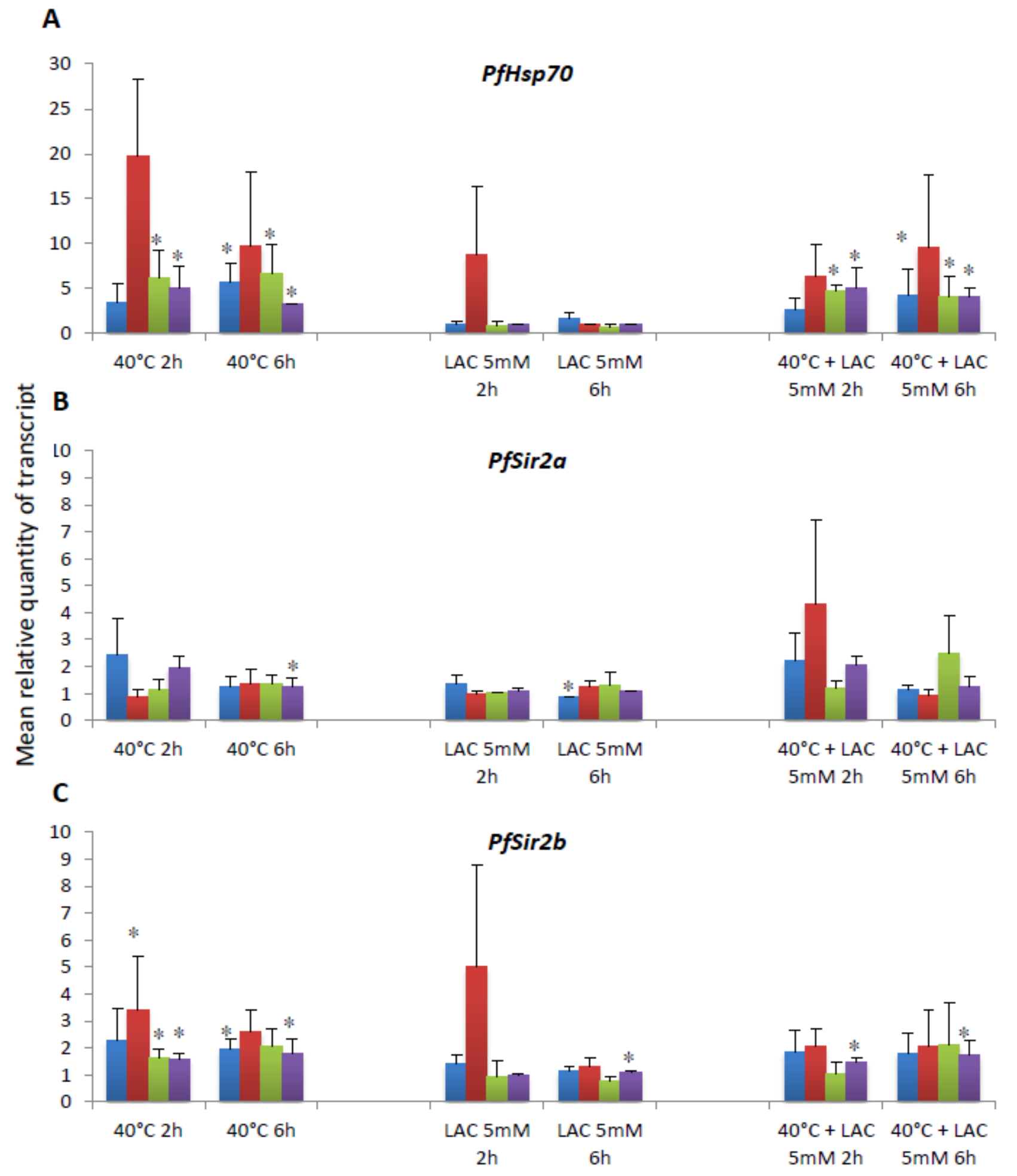

Figure 2

Increased expression of Hsp70, PfSir2a and PfSir2b in response to stress in trophozoites of P. falciparum. Relative quantity of transcripts for Hsp70 (A), Sir2a (B) and Sir2b (C) in parasites after stress treatments, 
relative to transcript quantities in a control culture. Blue bars, 3D7; red bars, ID3518; green bars, ID9775; purple bars, ID10668. Mean relative quantity is the pooled mean of $2(-\Delta \Delta \mathrm{Cq})$ from two biological replicate experiments in strains ID3518, ID9775 and ID10668, or three biological replicate experiments in 3D7. Error bars represent SEM from the pooled mean. ${ }^{*}, p<0.05$, Kruskal-Wallis test with Dunn's post test of the relative copy number $(\mathrm{RCN})$, derived from $2(-\triangle \mathrm{Cq})$.

A

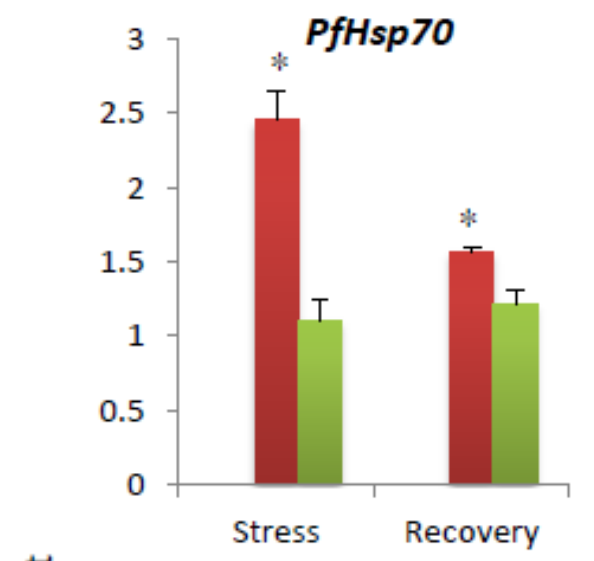

亳

$E$

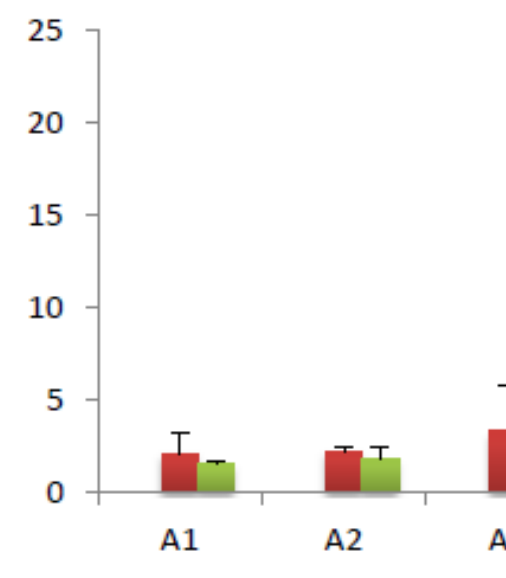

B

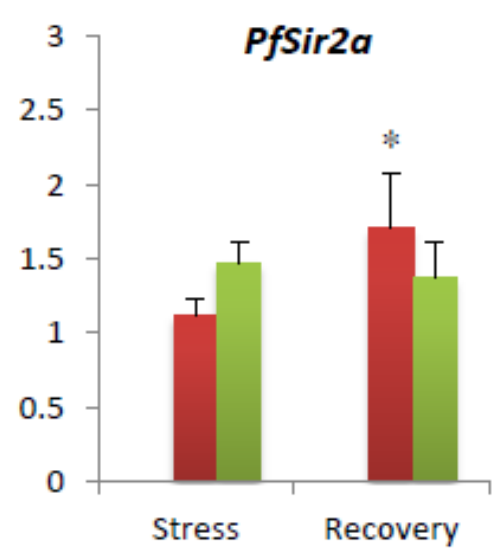

C

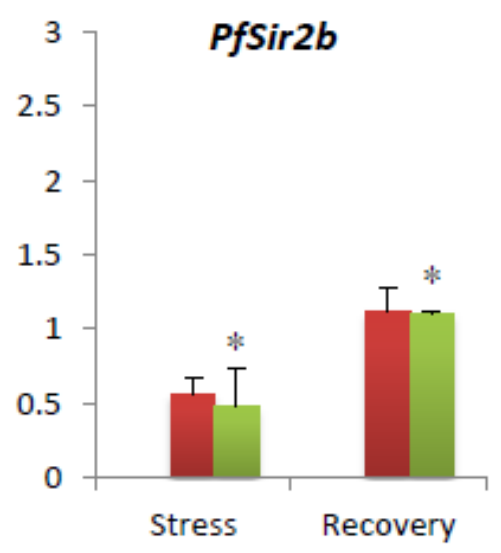

\section{Stress}

Var gene groups

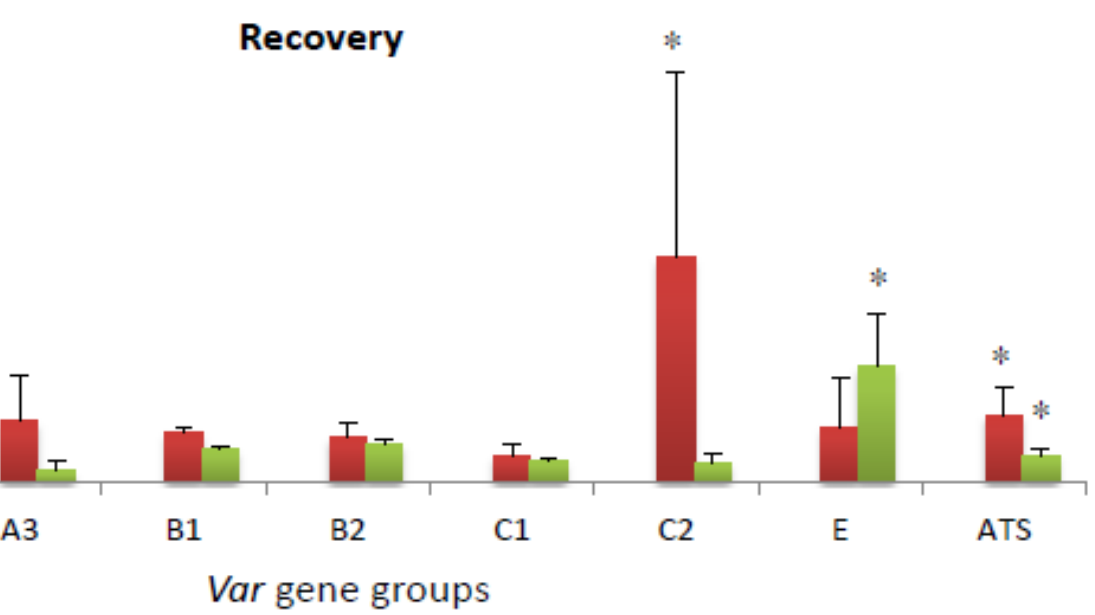

Figure 3 
Sirtuin and var gene transcription in ring-staged P. falciparum parasites after heat shock. Relative quantity of transcripts for Hsp70 (A), Sir2a (B) and Sir2b (C) in parasites immediately after $6 \mathrm{~h}$ of heat shock ('stress') and after a further $10 \mathrm{~h}$ of recovery ('recovery'), relative to transcript quantities in a control culture. Red bars, ID3518; green bars, ID9775. The corresponding relative quantities of var gene group transcripts are depicted in D. immediately after heat shock and $\mathrm{E}$. after $10 \mathrm{~h}$ of recovery. Mean relative quantity is the pooled mean of $2(-\Delta \Delta \mathrm{Cq})$ from two biological replicate experiments. Error bars represent SEM from the pooled mean. ${ }^{*}, p<0.05$, Kruskal-Wallis test with Dunn's post test of the relative copy number $(\mathrm{RCN})$, derived from $2(-\Delta \mathrm{Cq})$. 
A

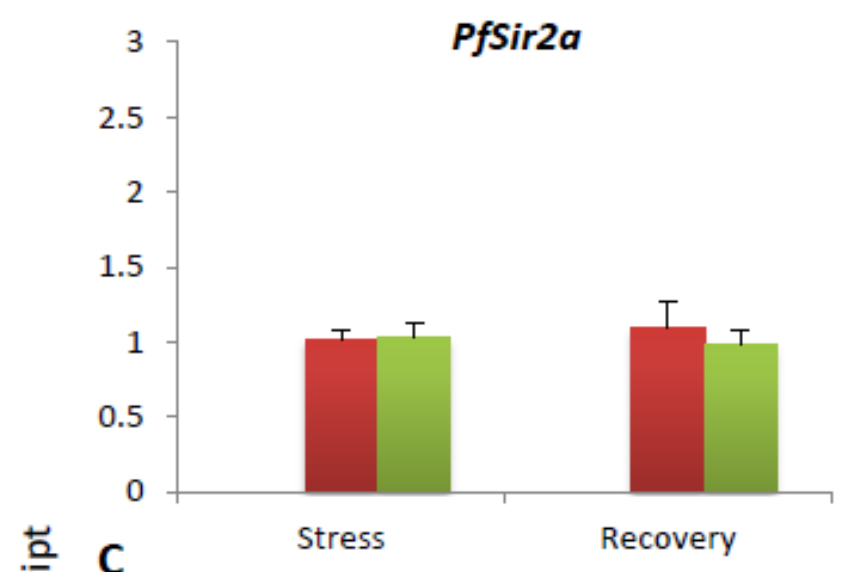

B

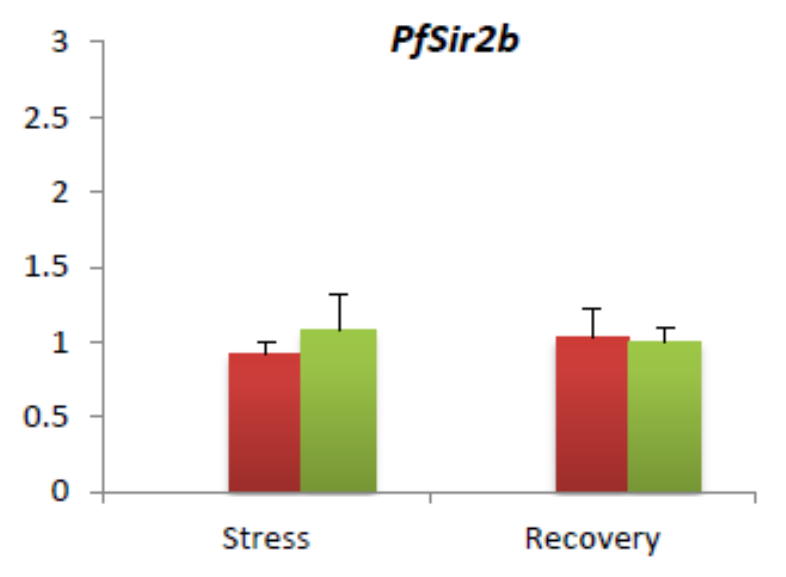

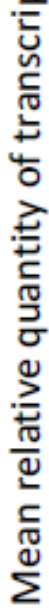

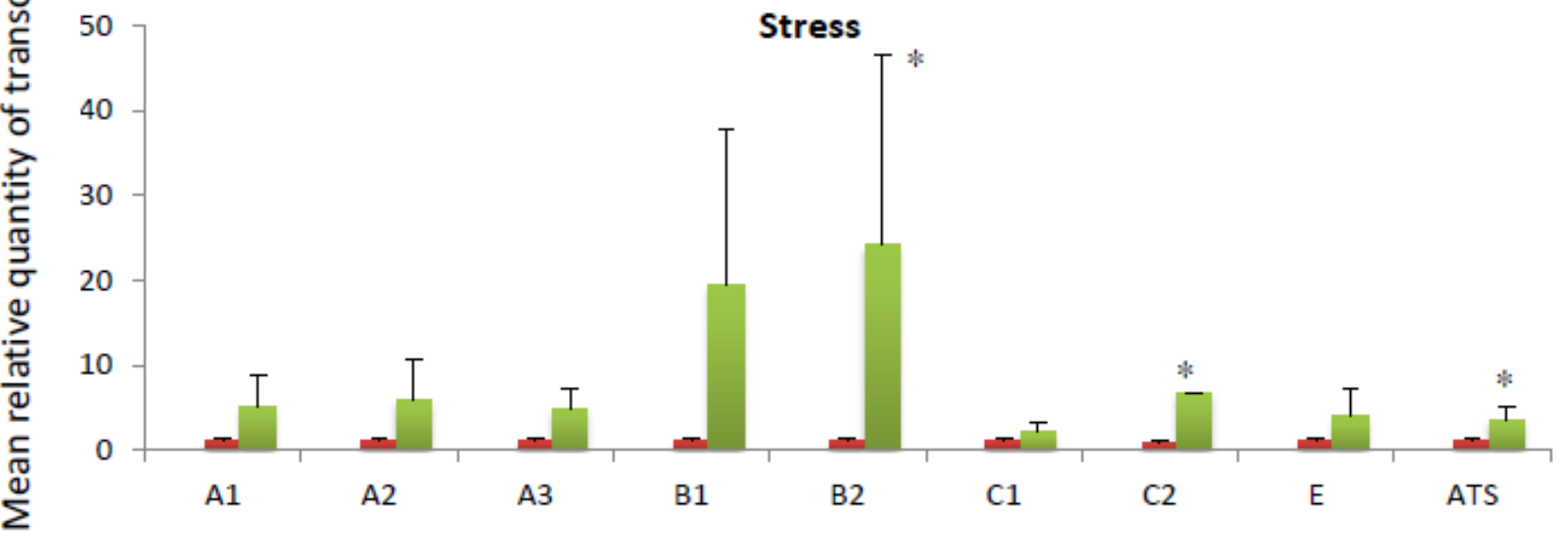

D

Var gene groups

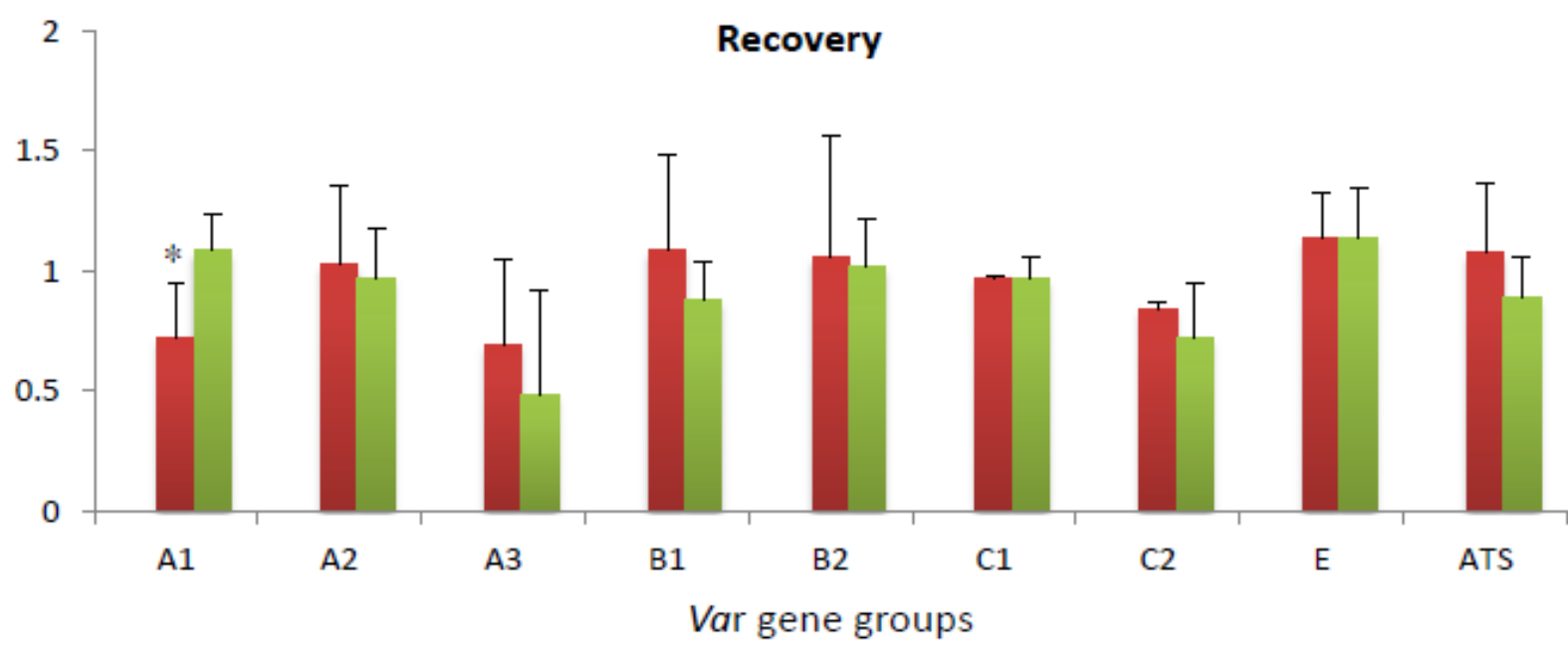

Figure 4

Sirtuin and var gene transcription in ring-staged P. falciparum parasites after exposure to elevated lactate. Relative quantity of transcripts for Sir2a (A) and Sir $2 b(B)$ in parasites immediately after $6 \mathrm{~h}$ of lactate exposure ('stress') and after a further $10 \mathrm{~h}$ of recovery ('recovery'), relative to transcript quantities in a control culture. Red bars, ID3518; green bars, ID9775. The corresponding relative quantities of var gene group transcripts are depicted in D. immediately after heat shock and e. after $10 \mathrm{~h}$ of recovery. Mean 
relative quantity is the pooled mean of $2(-\Delta \Delta \mathrm{Cq})$ from two biological replicate experiments. Error bars represent SEM from the pooled mean. ${ }^{*}, \mathrm{p}<0.05$, Kruskal-Wallis test with Dunn's post test of the relative copy number $(\mathrm{RCN})$, derived from $2(-\triangle \mathrm{Cq})$.

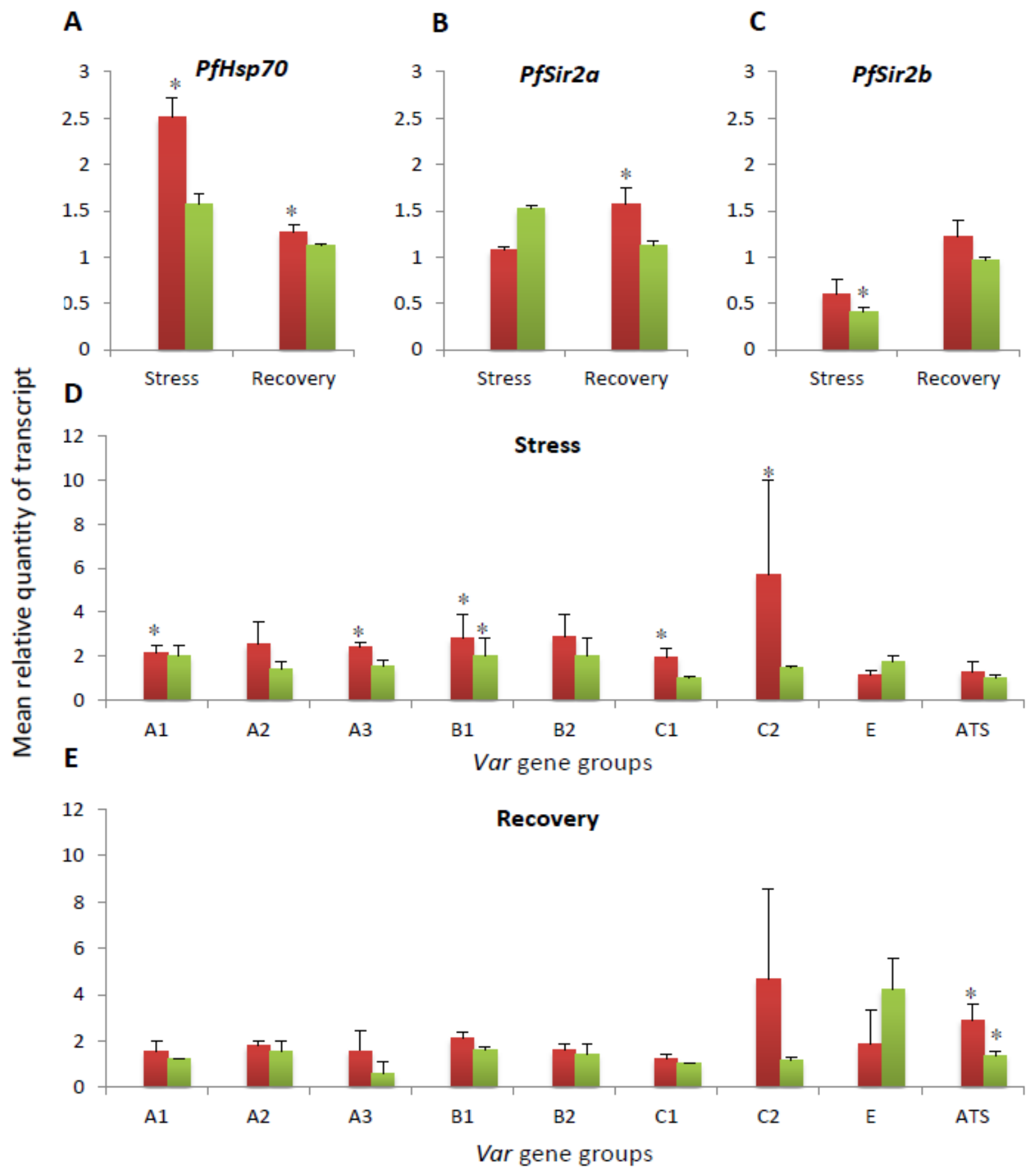

Figure 5

Sirtuin and var gene transcription in ring-staged P. falciparum parasites after heat shock combined with elevated lactate. Relative quantity of transcripts for Hsp70 (A), Sir2a (B) and Sir2b (C) in parasites 
immediately after $6 \mathrm{~h}$ of heat shock and lactate exposure ('stress') and after a further $10 \mathrm{~h}$ of recovery ('recovery'), relative to transcript quantities in a control culture. Red bars, ID3518; green bars, ID9775. The corresponding relative quantities of var gene group transcripts are depicted in $\mathrm{D}$. immediately after heat shock and $\mathrm{E}$. after $10 \mathrm{~h}$ of recovery. Mean relative quantity is the pooled mean of $2(-\Delta \Delta \mathrm{Cq})$ from two biological replicate experiments. Error bars represent SEM from the pooled mean. ${ }^{*}, p<0.05$, KruskalWallis test with Dunn's post test of the relative copy number $(\mathrm{RCN})$, derived from $2(-\Delta \mathrm{Cq})$.

\section{Supplementary Files}

This is a list of supplementary files associated with this preprint. Click to download.

- Supfig1.pdf

- SupplementaryTable1.docx 